\title{
Assessing the predictors of satisfaction with life of employees in Windhoek, Namibia
}

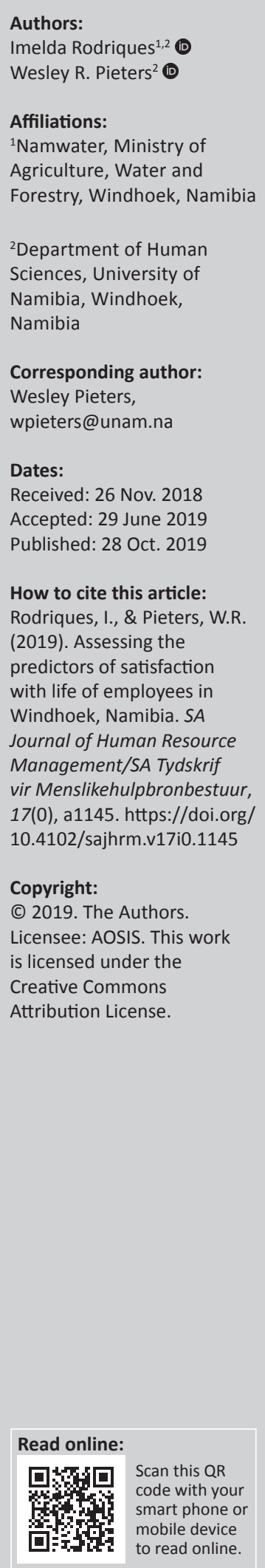

Orientation: Service delivering organisations are required to deliver quality services. It is of utmost importance to improve employees' satisfaction with life which is consistent with an organisation's effectiveness and efficiency.

Research purpose: This study investigated the impact of psychological capital (PsyCap) and general health on the satisfaction with life of employees of a parastatal and police officers in Windhoek, Namibia.

Motivation for the study: Poor service delivery and little regard for customer service are a result of unhealthy and dissatisfied employees spiralling into poor service delivery and customer dissatisfaction.

Research approach, design and method: A quantitative research approach (questionnaire) was used to collect data on the biographical details, PsyCap, general health and employees' satisfaction with life. The sample consisted of $(n=129)$ employees from a parastatal and $(n=152)$ employees of the Namibian Police Force.

Main findings: Psychological capital reported a positive relationship with satisfaction with life. General health reported a negative relationship with satisfaction with life. Hope and social dysfunction were found to be significant predictors of satisfaction with life.

Practical and managerial implications: Offering praise and recognition to hardworking employees enhances their self-efficacy, making them want to take on more challenging tasks while getting the needed guidance. Implementing stress management or wellness programmes helps employees cope better with job demands and improves employees' general health.

Contribution or value-add: Based on the literature, this is the first study carried out in Namibia on this topic, and it adds to existing knowledge within the customer service industry and paves the way for future researchers. This study will assist organisations in developing interventions aimed at keeping employees satisfied with life.

Keywords: psychological capital; general health; satisfaction with life; police officers; parastatal employees.

\section{Introduction}

Satisfaction with life has been defined as the overall assessment of an individual's quality of life based on one's own criteria (Lucas, Diener, \& Suh, 1996). This means that satisfaction with life is subjective; thus, different individuals will have different views about how satisfied they are with life. Sung-Mook and Effy (1994), on the contrary, defined satisfaction with life as an emotional reaction of individuals to their life consisting of work-time, spare time and time after work as well as expressing individual satisfaction about life. This means that an individual's satisfaction with life is impacted by both the individual's personal and work life. Thus, researches for the past three or more decades have concluded that job satisfaction is related to or spills over into satisfaction with life (Rode, 2004). The two ought to be related because work is the central life activity for most people, and much of working peoples' time is spent at their work.

According to Ioannou et al. (2015), employees who were dissatisfied with their job had low levels of general health as they experienced low vitality, low energy and increased physical pain. Increased job satisfaction was linked to increased physical and mental health (Ioannou et al., 2015). Given the importance of employee well-being at work, it is crucial that managers invest more attention towards improving the working conditions of their employees. 
Psychological capital (PsyCap) is an individual's positive psychological state of development. This development is characterised by: (1) having confidence (self-efficacy) to take on and put in the necessary effort to succeed at challenging tasks; (2) making a positive attribution (optimism) about succeeding now and in the future; (3) persevering towards goals and, when necessary, redirecting paths to goals (hope) to succeed; and (4) when overwhelmed by problems, difficulty, nourishment, bouncing back and even beyond (resilience) to attain success (Luthans, Youssef, \& Avolio, 2007). Luthans and Youssef (2004) defined PsyCap as a core psychological factor of positivity in general that goes beyond human and social capital to gain a competitive advantage through investment or development of 'who you are'. Based on this definition, at an individual level, PsyCap is a psychological resource that may fuel growth and performance. At an organisational level, PsyCap may provide leverage, return on investment and competitive advantage through improved employee performance (Luthans, Avolio, Walumbwa, \& Li, 2005).

General health entails a state of physical, mental and social well-being, not merely the absence of disease or infirmity, and this is a prerequisite for participation in a wide range of activities, for instance, work and education (WHO, 1948). Health in the workplace is an increasing fixation for organisations. The fixation is attributed to the positive links that have been found between the well-being of employees, productivity and performance (Fenton, Roncancio, Sing, Sandhra, \& Carmichael, 2014). These links are also relevant to state-owned enterprises within Namibia.

Abolghasemi and Varaniyab (2010) showed that both resilience and perceived positive stress are positively related to life satisfaction, suggesting that an increase of resilience and a decrease of stress lead to increased life satisfaction. Increased life satisfaction enables an individual to feel better and to develop resources for coping with life. Evidence supports a relationship between the frequency of depression and anxiety or poor mental health with low job quality and work stress. This is the main factor as to why employers engage in interventions that promote health and encourage workplace well-being (Fenton et al., 2014).

The introduction of the State-Owned Enterprises Act (2 of 2006) of Namibia and its governance structures was aimed at ensuring that the performance of state-owned enterprises is maximised (Amunkete, 2015). Despite these efforts by the government to regulate state-owned enterprises and improve performance, to date the poor performance of state-owned enterprises remains a pertinent issue in Namibia. Stateowned enterprises need to take a positive approach that will recognise and leverage human resources for contribution to sustainable growth, competitive advantage and performance if they are to improve performance (Amunkete, 2015).

The Namibian water services parastatal, Namwater, where this study was conducted, has a high degree of autonomy, although its charges and sourcing of external financing are approved by the Namibian government (Ndokosho, 2006). The parastatal has been compounded by poor financial performance. This might lead to underinvestment and a weakening of infrastructure, and this can result in inefficiency of employees and declined service quality.

Similarly, for the police force (where the study was conducted), their work has been viewed as one of the most tiring and stressful jobs in the world (Lu, Liu, Sui, Guoyuan, \& Wang, 2015). It is stressful because of shift work, threats of violence, excessive paperwork, confrontational contact with the public, the militaristic nature of the work and having to deal with a bureaucratic organisational structure. According to the Namibian Police Act (19 of 1990), the function of the police force shall be the preservation of the internal security of Namibia, the maintenance of law and order, the investigation of any offence or alleged offence, the prevention of crime and the protection of life and property. In some cases, the police force fails to fulfil their functions (Haidula, 2018). The police force may be perceived as unreliable and not meeting the objectives stipulated in their mandate. This can be seen in the way members of the public try to take justice into their own hands.

\section{Purpose}

Studies related to PsyCap in Namibia are still novel and few studies (Amunkete, 2015; Ndokosho, 2006) have been undertaken into PsyCap and other related variables. Most studies with a similar focus were conducted outside the Namibian context. The researchers did not find any literature regarding the relationship between PsyCap, general health and satisfaction with life. According to the researchers' knowledge, this will also be the first study to investigate the predictors of satisfaction with life in the Namibian context. This study aims at investigating the relationship between these variables and the impact that PsyCap and general health have on the life and job satisfaction of employees of in-service delivering organisations. This study further aims to influence the perception of employers concerning the understanding and importance of life and job satisfaction in the organisation.

\section{Literature review}

\section{Psychological capital and satisfaction with life}

Life satisfaction can be partly viewed as the result of satisfaction with various life domains, which range from satisfaction within specific life domains to overall life satisfaction (Rode, 2004). Some scholars primarily attribute satisfaction with life to personality factors, and genetic and social-cognitive factors like goal-directed activity, selfefficacy, outcome expectations, environmental support and resources (Mafini, Surujlal, \& Dhurup, 2012). Some people are born more optimistic and satisfied with life; some people have a certain personality disposition to be more satisfied and others are more or less satisfied depending on what they have or what is available in their environment in relation to what they regard as important (Mafini et al., 2012). 
Psychological capital is derived from the POB (positive organisational behaviour) foundation and criteria (Luthans et al., 2007). Psychological capital is a higher order core construct that fits into the various POB abilities of: (1) a construct being theory and evidence-based; (2) the construct needs to be positively oriented; (3) it should be validly and reliably measurable; (4) it needs to be open to development and management; and (5) lastly the construct should be related to desired and measurable work attitudes, behaviours and performance criteria for it to be included in $\mathrm{POB}$ (Luthans \& Youssef-Morgan, 2017). Luthans (2002, p. 59) defined $\mathrm{POB}$ as the study and application of positively oriented human resource strengths and psychological capacities that can be measured, developed and effectively managed for performance improvement in today's workplace'.

Psychological capital is a multidimensional construct associated with four positive psychological resources: hope, self-efficacy, resilience and optimism (Luthans \& YoussefMorgan, 2017). These resources share a sense of control, intentionality and agentic goal pursuit. They also share the common theme of positive appraisal of circumstances and probability for success based on motivated effort and perseverance (Luthans \& Youssef-Morgan, 2017). These impact an individual's satisfaction with life in relation to how motivated and persistent they are. According to Avey, Wernsing and Mhatre (2011), self-efficacious individuals tend to have stronger beliefs in their abilities and capacities to perform various tasks that increase effort and persistence in the pursuit of their goals. High levels of hope help individuals generate the required agentic striving and pursue multiple pathways towards attainment of goals that lead to greater instances of successful outcomes. High levels of optimism help individuals maintain a positive perspective and expectations with regard to the outcomes. High levels of resilience assist individuals to overcome adversity and 'bounce back' from episodes of potential or actual failure. These are the features that characterise productive employees in the workforce, and this spills over to their job and life satisfaction (Avey et al., 2011).

This study indicated that PsyCap has a positive relationship with performance and job satisfaction; it is also a better predictor of these outcomes than its individual components (Luthans, Avolio, Avey, \& Norman, 2007). Findings indicate that operating conditions and the nature of work are significantly and negatively related to psychological distress (Aazami, Shamsuddin, Akmal, \& Azami, 2015). They further stressed that these findings indicated that when work was perceived to be meaningful, important, enjoyable, and employees not being overwhelmed with paperwork, they experienced less psychological distress and higher levels of job satisfaction. Psychological capital is believed to trigger cognitive, affective, conative and social mechanisms in people, leading to happiness and well-being. Happy and healthy people tend to be more satisfied with their lives. Avey, Luthans, Smith and Palmer (2010) stated that employees' PsyCap reinforces the potential value of taking different perspectives, appraising situations and circumstances in more positive, opportunistic, adaptive and promotion-focussed ways that enhance their well-being.

\section{General health and satisfaction with life}

Satisfaction with life is the cognitive component of subjective well-being. Health of employees within the workplace has been getting significant attention over the years. This attention is linked to positive outcomes such as increase in performance and productivity (Fenton et al., 2014). The literature indicates that there is a large number of interventions aimed at raising physical activities, improved nutrition and reduced smoking at work. The common purpose of these interventions is to improve the quality of life and the productivity of workers and to reduce future health care cost associated with cardiovascular or non-transmittable diseases (Fenton et al., 2014).

There are hedonic and eudemonic aspects of well-being. Hansen (2012) argued that the hedonic aspect of well-being refers to subjective emotions such as happiness and the experience of pleasure, and is characterised by the presence of positive moods and the absence of negative moods. The eudemonic aspect of well-being is more cognition based and focussed on the motivation individuals possess to achieve their goals, and thereby contributes to positive feelings.

Thus, focussing on well-being at work presents a valuable opportunity to benefit not only organisations but societies as well, which helps working individuals to feel happy, competent and satisfied in their roles. Employees who achieve this are likely to be more creative, loyal and provide better customer satisfaction (Hansen, 2012).

The study by Fenton et al. (2014) found a relationship between the frequency of depression and anxiety or poor mental health with low job quality and work stress. These are the leading factors as to why employers engage in interventions that promote health and encourage workplace well-being (Fenton et al., 2014). Reasons for these interventions have to do with employers' need to increase employee morale or job satisfaction and reduce the spillover effect that ill health has on one's co-workers, supervisors or family members (Fenton et al., 2014). They further stated that research has identified some mental and physical health promotion programmes classified as employee assistance programmes (EAPs) or health promotion programmes (HPPs) and assessed their effectiveness. The EAPs are wellness interventions in which employees receive short-term counselling for issues such as family problems or substance abuse. Health promotion programmes constitute a more traditional approach that offers educational interventions aimed at supporting the development and maintenance of health-related behaviours conducive for the well-being of the overall workforce (Fenton et al., 2014). The principle behind EAP's and HPP's is that they improve health, and this reduces absenteeism and costs that leads to life satisfaction. 
The study by Aazami et al. (2015) reported a relationship between satisfaction with life and general health, and wellbeing or health in the workplace. Hence, there is potential of gains for organisations from intervening in employees' health, which outweighs the costs of not intervening. This is similar with what other researchers found in their studies. Aazami et al. (2015) found that satisfaction with the nature of one's work significantly predicts sleep disorders, headaches and gastrointestinal problems. They also found that work stress conditions are associated with the development of psychosomatic complaints. Poor working conditions are directly associated with job dissatisfaction and sleep shortness. Well-being was also found to be related to a wide range of positive outcomes such as physical and psychological health and satisfaction with important life domains. Faragher, Cass and Cooper (2018) stated that employees' level of job satisfaction is a key factor influencing workers' health. Thus, there is a need for employers to support and promote the health and well-being of their employees, as well-being is a broad category of phenomena that includes people's emotional responses, domain satisfaction and global judgements of life satisfaction (Hansen, 2012). Interventions to support health promotion in the workplace can reduce the cost of health care for employees which benefits organisations that have to pay the health insurance of their employees (Fenton et al., 2014). Furthermore, well-being plays a central role in creating flourishing societies focussing on well-being at work, thereby presenting a valuable opportunity to benefit societies as this helps working individuals to feel happy, competent and satisfied in their roles.

\section{Psychological capital and general health}

Psychological capital as representative of positive workrelated psychological resources is related to measures of well-being, and it also adds a small but significant variance to well-being over time (Avey et al., 2010). Thus, positive resources such as employees' PsyCap may lead to the desirable outcome of their psychological well-being over time. Briefly, high levels of PsyCap lead to greater number of positive outcomes, and this intermediary mechanism perpetuates a process of optimal psychological functioning, which is well-being. The relationship between PsyCap and general health is supported by the conservation of resources (COR) theory (Avey et al., 2010). Psychological capital as a whole is a better predictor of job satisfaction and performance than each of the components of PsyCap (Luthans et al., 2007).

Based on the aforementioned literature, the following hypotheses were developed:

Hypothesis 1: Psychological capital has a positive relationship with satisfaction with life.

Hypothesis 2: General health has a negative relationship with satisfaction with life.

Hypothesis 3: Psychological capital and general health are significant predictors of satisfaction with life.

\section{Method Research approach}

Making use of the quantitative research method, a questionnaire was used to collect the biographical data of employees, PsyCap, general health and satisfaction with life. The researchers approached a parastatal that provides services to clients and the Namibian Police Force. These organisations were approached at random. These organisations had to provide approval for research to be carried out with their employees. Research was only carried out upon receiving approval for conducting research from the organisations. Participants were approached; the purpose and how the data will be used were explained. After employees were informed of their rights to confidentiality and anonymity, those who were interested signed the consent form and completed the questionnaire. The participants were given a few days to complete the questionnaire and the researchers collected the questionnaires. Participants had the option to withdraw from the study at any time or not to participate without fear of discrimination, victimisation or negative consequences.

\section{Participants}

Making use of non-probability sampling, participants who were available and willing were included from the Namibian Police Force and Namwater in Windhoek. From Namwater, 129 participants $(45.9 \%)$ and 152 participants from the Namibian Police Force (54.1\%) took part in the study. In total, 300 questionnaires were distributed, and 281 questionnaires were returned (response rate 93.7\%). The distribution between males and females was fairly even with $48 \%$ females $(n=135)$ and $52 \%$ males $(n=146)$. Many participants were aged between 24 and 28 years $(21 \%, n=59)$, and a small portion were aged below 24 years of age $(5.3 \%, n=18)$. Participants had worked for $7-8$ years $(18.9 \%, n=53)$, and only 18 participants $(6.4 \%)$ had worked for $11-15$ years. With regard to marital status, $62.6 \%(n=176)$ of the participants were single, and only two participants $(0.7 \%)$ were widowed. Majority of the sample $(42 \%, n=118)$ had $1-2$ dependents, while $2.5 \%$ of the participants $(n=7)$ had 10 or more dependents. Regarding qualifications, 69 participants (24.6\%) had obtained honours degrees as their highest qualification, and $4.6 \%(n=13)$ had a grade 12 certificate as their highest qualification. The remaining data of the biographical information are presented in Table 1.

\section{Measuring instruments}

A demographic questionnaire developed by the researchers was used to obtain information about participants' characteristics, including sex, age, tenure, marital status, number of dependents and highest qualification obtained.

Psychological capital was measured using the PsyCap scale developed by Luthans et al. (2007). This scale evaluated employees' level of PsyCap by focussing on the four PsyCap components or dimensions: hope, self-efficacy, resilience and optimism. The questionnaire consists of 24 items 
TABLE 1: Biographical details of sample $(n=281)$.

\begin{tabular}{|c|c|c|c|}
\hline Item & Category & Frequency & Percentage \\
\hline \multirow[t]{2}{*}{ Gender } & Male & 146 & 52.0 \\
\hline & Female & 135 & 48.0 \\
\hline \multirow[t]{8}{*}{ Age } & Below 24 years & 15 & 5.3 \\
\hline & $24-28$ years & 59 & 21.0 \\
\hline & 29-31 years & 47 & 16.7 \\
\hline & $32-35$ years & 56 & 19.9 \\
\hline & $36-40$ years & 44 & 15.7 \\
\hline & $41-45$ years & 27 & 9.6 \\
\hline & $46-50$ years & 18 & 6.4 \\
\hline & 51 years and above & 15 & 5.3 \\
\hline \multirow[t]{8}{*}{ Tenure } & Less than a year & 27 & 9.6 \\
\hline & $1-2$ years & 24 & 8.5 \\
\hline & $3-4$ years & 40 & 14.2 \\
\hline & $5-6$ years & 44 & 15.7 \\
\hline & $7-8$ years & 53 & 18.9 \\
\hline & $9-10$ years & 31 & 11.0 \\
\hline & $11-15$ years & 18 & 6.4 \\
\hline & 16 years and more & 44 & 15.7 \\
\hline \multirow[t]{4}{*}{ Marital status } & Single & 176 & 62.6 \\
\hline & Married & 96 & 34.2 \\
\hline & Divorced & 7 & 2.5 \\
\hline & Widowed & 2 & 0.7 \\
\hline \multirow[t]{6}{*}{ Dependents } & None & 59 & 21.0 \\
\hline & $1-2$ & 118 & 42.0 \\
\hline & $3-4$ & 64 & 22.8 \\
\hline & $5-6$ & 25 & 8.9 \\
\hline & $7-9$ & 8 & 2.8 \\
\hline & 10 and more & 7 & 2.5 \\
\hline \multirow{6}{*}{$\begin{array}{l}\text { Highest } \\
\text { qualification } \\
\text { obtained }\end{array}$} & Grade 12 & 13 & 4.6 \\
\hline & Certificate & 58 & 20.6 \\
\hline & Diploma & 40 & 14.2 \\
\hline & Degree & 60 & 21.4 \\
\hline & Honours degree & 69 & 24.6 \\
\hline & Masters & 40 & 14.2 \\
\hline Total & & 281 & 100.0 \\
\hline
\end{tabular}

comprising four scales with six items per scale. These six items measure an individual's ability to persevere towards goals and redirect paths to goals to succeed (hope). For example, 'Right now I see myself as being pretty successful at work'. The other six items measure an individual's ability to have confidence to take on and put in the necessary effort to succeed at challenging tasks (efficacy), for example, 'I feel confident helping to set targets or goals in my work area'. The following six items measure an individual's ability to sustain and bounce back when affected by problems and adversity to attain success (resilience), for example, 'I usually take stressful things at work in stride'. The last six items measure an individual's ability to make a positive attribution and expectation about succeeding now and in the future (optimism), for example, 'I always look on the bright side of things regarding my job' (Luthans et al., 2007). The response format of the questionnaire includes response choices in a six-point Likert-type scale, with $1=$ strongly disagree, 2=disagree, $3=$ somewhat disagree, 4=somewhat agree, $5=$ agree and $6=$ strongly agree. In a study conducted in the Namibian context, a reliability of $\alpha=0.97$ for the PsyCap questionnaire was found (Amunkete, 2015). In the same study, the following reliabilities were found for the four
PsyCap constructs: $\alpha=0.84$ (self-efficacy), $\alpha=0.81$ (hope), $\alpha=0.63$ (resilience) and $\alpha=0.65$ (optimism).

To measure the general health of employees, the general health scale developed by Goldberg and Hillier (1979) was used. The scale consists of 28 items consisting of three subscales: somatic symptoms, for example, 'Been feeling run down and out of sorts?'; anxiety and insomnia, for example, 'Lost much sleep over worry?'; and social dysfunction (C scale), for example, 'Been managing to keep yourself busy and occupied?' (Goldberg \& Hillier, 1979). The questionnaire aims at assessing the level of general health on a four-point Likert scale (from 1 = more than usual to $4=$ much less usual). The Cronbach's alpha found for the General Health Questionnaire (GHQ) reliability is of 0.85 (Haidula, Shino, Plattner, \& Feinstein, 2003). The reliability for the general health subscales was the following: somatic symptoms $\alpha=$ 0.71 , anxiety and insomnia $\alpha=0.7$, social dysfunction $\alpha=$ 0.81 and depression $\alpha=0.88$ (Haidula et al., 2003).

Satisfaction with life was measured using the satisfaction with life scale developed by Diener, Emmons, Larsen and Griffin (1985) to describe how employees view their work and feel about life in general. This is a five-item scale designed to measure global cognitive judgements of one's life satisfaction (Diener et al., 1985). One of the sample item is: 'In most ways my life is close to my ideal'. With a response format of a seven-point Likert scale ranging from 1 = strongly agree to 7 = strongly agree, the reliability of the instrument is $\alpha=0.86$ (Galanakis, Lakioti, Pezirkianidis, Karakasidou, \& Stalikas, 2017).

\section{Design}

The researchers used the quantitative approach, which is a process that is systematic and objective in its ways of using numerical data from a selected sample of the population, so that the findings are generalised to the population that is being studied (Maree \& Pietersen, 2007). This method was used to collect information on PsyCap, general health and employees' satisfaction with life. The research is descriptive in nature, and information was collected using questionnaires that will allow participants to rate their perceptions on a scale to describe PsyCap, general health and satisfaction with life. The researchers also used correlation to test for relationships between these variables and regression to test for predictability.

\section{Analysis}

The data collected were analysed using the Statistical Package of Social Sciences version 24 (SPSS, 2016). This allowed the researcher to use graphs, pie charts and tables to present the data and to make correlations between variables. Descriptive statistics, a data analysis technique used to describe demographic characteristics, behaviours or outcomes of a study (Beins \& McCathy, 2012), was used to convey the biographical results of the study. Statistical Package of Social Sciences' reliability analysis technique, 
specifically the Cronbach's alpha technique, was used to test the consistency of instruments used in the study. This ensured that the results produced by the instruments were reliable. The researchers also made use of inferential statistics - the use of likelihood, or probability, to assess whether an event is common or unusual (Beins \& McCathy, 2012). The Pearson product moment (Pearson $=r$ ) was used to determine whether there was an association between the different variables of the study. Making use of stepwise multiple regression, the predictability of the dependent variable was used with the inclusion of the independent variable.

\section{Ethical considerations}

Permission was obtained from the Inspector General of the Ministry of Safety and Security and the Head of Human Resources of Namwater.

\section{Results \\ Descriptive statistics and correlations}

The data were analysed to determine the means, SDs, Cronbach's alpha and correlations that are presented in Table 2.

This study reported a mean of 28.74 for self-efficacy and an SD of 5.29. Hope reported a mean of 28.86 and an SD of 4.69. Somatic symptoms reported a mean of 11.56 and SD of 3.77 . Anxiety or insomnia reported a mean of 23.69 and an SD of 6.98. Social dysfunction reported a mean of 8.93 and SD of 3.18. Satisfaction with life reported a mean of 23.12 and SD of 6.56 .

Cronbach's alpha was also reported for self-efficacy of $\alpha=0.80, \alpha=0.79$ for hope, $\alpha=0.74$ for somatic symptoms, $\alpha=87$ for anxiety or insomnia, $\alpha=0.79$ for social dysfunction and $\alpha=0.78$ for satisfaction with life. Optimism and resilience did not meet the expected reliability cut-off of $\alpha=0.70$ and were excluded from further analysis in the study.

Making use of the Pearson correlation coefficient, the following relationships were found between the variables. Psychological capital, self-efficacy, had a positive correlation with PsyCap-hope ( $r=0.58, p<0.05$; large effect), and a negative correlation with somatic symptoms (general health) $(r=-0.23, p<0.05$; small effect), with anxiety or insomnia (general health) $(r=-0.26, p<0.05$; small effect), with social dysfunction (general health) $(r=-0.18, p<0.05$; small effect) and a positive correlation with satisfaction with life $(r=0.27$, $p<0.05$; small effect).

PsyCap-hope reported a negative relationship with somatic symptoms (general health) ( $r=-0.26, p<0.05$; small effect), with anxiety or insomnia (general health) $(r=-0.38, p<0.05$; medium effect), with social dysfunction (general health) $(r=-0.21, p<0.05$; small effect) and a positive relationship with satisfaction with life $(r=0.31, p<0.05$; medium effect).

Somatic symptoms (general health) reported a positive correlation with anxiety or insomnia (general health) $(r=0.64, p<0.05$; large effect), with social dysfunction (general health) ( $r=0.31, p<0.05$; medium effect) and a negative correlation with satisfaction with life $(r=-0.26$, $p<0.05$; small effect).

Anxiety or insomnia (general health) reported a positive correlation with social dysfunction (general health) $(r=0.54$, $p<0.05$; large effect) and a negative correlation with satisfaction with life $(r=-0.33, p<0.05$; medium effect).

Social dysfunction (general health) reported a negative relationship with satisfaction with life $(r=-0.30, p<0.05$; medium effect).

\section{Multiple regression analyses}

To test hypothesis 3, multiple regression analyses were performed. In the first model, PsyCap (hope) was inserted into the relationship with satisfaction with life being the dependent variable. In model 2, anxiety or insomnia and social dysfunction (general health) were included into the model.

In Table 3, it can be seen that when PsyCap (hope) was introduced into model 1 , it produced a significant model $\left(F_{(1,259)}=27.64 ; p<0.00\right)$ and accounts for about $10 \%$ of the variance $(0.096 \%)$. It appears that PsyCap (hope) $(\beta=0.31$; $t=5.26 ; p<0.00)$ is a significant predictor of satisfaction with life. Anxiety or insomnia and social dysfunction (general health) were introduced in model 2 to produce a significant

TABLE 2: Descriptive statistics, reliability and Pearson correlation coefficient.

\begin{tabular}{|c|c|c|c|c|c|c|c|c|c|}
\hline \multirow[t]{2}{*}{ Item } & \multicolumn{2}{|c|}{ Total } & \multirow[t]{2}{*}{$\alpha$} & \multirow[t]{2}{*}{1} & \multirow[t]{2}{*}{2} & \multirow[t]{2}{*}{3} & \multirow[t]{2}{*}{4} & \multirow[t]{2}{*}{5} & \multirow[t]{2}{*}{6} \\
\hline & Mean & SD & & & & & & & \\
\hline 1. Psycap-SE & 28.74 & 5.29 & 0.80 & - & - & - & - & - & - \\
\hline 2. Psycap-H & 28.86 & 4.69 & 0.79 & $0.58 \%$ & - & - & - & - & - \\
\hline 3. GH-SS & 11.56 & 3.77 & 0.74 & $-0.23 *$ & $-0.26^{*}$ & - & - & - & - \\
\hline 4. GH-AI & 23.69 & 6.98 & 0.87 & $-0.26 *$ & $-0.38 * \dagger$ & $0.64 \ddagger$ & - & - & - \\
\hline 5. GH-SD & 8.93 & 3.18 & 0.79 & $-0.18^{*}$ & $-0.21 *$ & $0.31 * \dagger$ & $0.54 t$ & - & - \\
\hline 6. SWL & 23.12 & 6.56 & 0.78 & $0.27 *$ & $0.31 * \dagger$ & $-0.26^{*}$ & $-0.33 * \dagger$ & $-0.30 * \dagger$ & - \\
\hline
\end{tabular}

PsyCap, psychological capital; PsyCap-SE, psychological capital (self-efficacy); PsyCap-H, psychological capital (hope); GH, general health; GH-SS, general health (somatic symptoms); GH-Al, general health (anxiety or insomnia); GH-SD, general health (social dysfunction); SWL, satisfaction with life.

*, Statistically significant: $p \leq 0.05$.

$*+$, Practically significant correlation (medium effect): $0.30 \leq r \leq 0.49$.

$\$$. Practically significant correlation (large effect): $r \geq 0.50$. 
TABLE 3: Multiple regression with satisfaction with life being the dependent variable and hope (psychological capital), anxiety or insomnia and social dysfunction (general health) the independent variables.

\begin{tabular}{|c|c|c|c|c|c|c|c|c|}
\hline Model & & & $\begin{array}{l}\text { Standardised } \\
\text { coefficients }(\beta)\end{array}$ & $t$ & $p$ & $F$ & $R^{2}$ & $\Delta R^{2}$ \\
\hline \multicolumn{9}{|l|}{1} \\
\hline (Constant) & 10.57 & 2.42 & - & 4.37 & 0.00 & 27.64 & 0.31 & 0.096 \\
\hline PsyCap_H & 0.44 & 0.08 & 0.31 & 5.26 & $0.00 *$ & & & \\
\hline 2 & & & & & 0.00 & 17.29 & 0.41 & 0.168 \\
\hline (Constant) & 20.97 & 3.31 & - & 6.33 & 0.00 & & & \\
\hline PsyCap_H & 0.30 & 0.09 & 0.22 & 3.51 & $0.01 * *$ & & & \\
\hline GH_Al & -0.15 & 0.07 & -0.16 & -2.21 & 0.28 & & & \\
\hline GH_SD & -0.34 & 0.14 & -0.17 & -2.44 & $0.02 *$ & & & \\
\hline
\end{tabular}

$t$, test; $p$, probability value; $F$, overall significance; $R^{2}$, percentage variance explained; $\Delta R^{2}$, change in percentage variance explained; $B$, regression coefficient; $\mathrm{SE}$, standard error. $*, p \leq 0.05 ; * *, p \leq 0.01$.

model $\left(F_{(3257)}=17.29 ; p<0.00\right)$ and account for about $16.8 \%$ of the variance. It appears that PsyCap (hope) $(\beta=0.22 ; t=3.51$; $p<0.01)$ and social dysfunction $(\beta=-0.17 ; t=-2.44 ; p<0.02)$ are significant predictors of satisfaction with life. Anxiety or insomnia $(\beta=-0.16 ; t=-2.21 ; p<0.28)$ was found not to be a significant predictor of satisfaction with life.

\section{Discussion}

\section{Outline of the results}

Hypothesis 1 of the study investigated the relationship between PsyCap and satisfaction with life. The results of this study support hypothesis 1 . This study found a positive (small and medium) relationship between PsyCap (self-efficacy; hope) and satisfaction with life. If employees regard their abilities to be sufficient to execute their work duties, they would try harder and apply continuous effort to achieve their goals. Being hopeful will increase the employees' ability to continue working hard, believing in a positive outcome. Mafini et al. (2012) indicated that personality factors also impact the levels of satisfaction of life. Employees who are able to achieve at work enhance their self-efficacy and levels of hope. These employees are likely to also experience a higher level of job satisfaction and satisfaction with life (Rode, 2004).

The study found a negative relationship between general health (somatic symptoms, anxiety or insomnia and social dysfunction - negative dimensions) and satisfaction with life. The results support hypothesis 2 of the study. When employees suffer from physical ailments, are unable to sleep well or worry about things, they tend to have a negative interaction with others and they are likely to be less satisfied with life. Ioannou et al. (2015) indicated that when employees are experiencing ill health, they are less likely to perform well at work or experience job satisfaction. When employees are experiencing higher levels of well-being, they are likely to experience pleasure in their jobs, become productive and experience higher levels of satisfaction with life (Fenton et al., 2014). The findings of this study are also supported by Abolghasemi and Varaniyab (2010), who found that positive stress is positively related to satisfaction with life. Somatic symptoms, anxiety or insomnia and social dysfunction are regarded as negative aspects of health, negatively impacting the satisfaction of life. Fenton et al. (2014) indicated that EAPs and HPPs are interventions that improve the health and wellbeing of employees, improve their general health, reduce absenteeism and most likely increase employees' satisfaction with life.

Based on the results of the study, PsyCap and general health are significant predictors of satisfaction with life. The results of this study support hypothesis 3. When employees are confident about their abilities, are more hopeful, experience good physical health, sleep well, are not too worried about matters beyond their control and are able to interact with customers and others well, they experience a higher level of satisfaction with life (Abolghasemi \& Varaniyab, 2010; Fenton et al., 2014; Ioannou et al., 2015).

\section{Practical implications}

Self-efficacy shares some similarity with the agency (willpower) component of hope (Herbert, 2011). On the contrary, hopeful thoughts are the beliefs that one can find pathways to desired goals and become motivated to use those pathways (Herbert, 2011). Self-efficacy has been conceptualised as individuals' beliefs in their capabilities to mobilise motivation, cognitive resources and agency to exert control over a given event (Hamill, 2003). Thus, self-efficacy can be said to be an antecedent of hope. Organisations need to provide employees with an environment that allows them to set goals that are inspiring, slightly challenging and realistic. With the guidance of supervisors, employees should be assisted to set a career path for themselves and be provided with the necessary resources to achieve these goals. When employees achieve these goals, they improve their self-belief and desire to achieve more challenging goals. In this way, employees become more determined to achieve goals at work and in turn the productivity of organisations improve.

Supervisors and managers may also praise and recognise hard-working employees who achieve organisational goals. Praise and recognition enhance employees' self-efficacy and motivate them to want to do more at work.

When employees have a career plan or path, they are likely to also become more hopeful about the future. Believing in a better future may ensure that employees will work harder and engage in work-related activities. By working harder, 
employees could be promoted to a better position within the organisation, with better benefits and hopefully a higher salary. Mohiadin and Mostafai (2012) indicated that when organisations are able to improve the self-efficacy of employees, they are likely to also enhance the employees' satisfaction with life.

Self-efficacy can also be enhanced through training and development. When employees lack the necessary skills to execute their work duties, they are likely to perform poorly and have low self-efficacy. Empowering employees with the necessary skills would increase their ability to perform better and perceive themselves as more competent at work.

Providing employees with medical aid benefits allows them to visit trained health care providers and get necessary treatment. Considering that medical aid is an important benefit to employees, this also enhances their levels of job satisfaction and satisfaction with life. Organisations, through their wellness offices, can invite health care service providers to demonstrate how to exercise effectively, discuss how to improve and maintain a healthy lifestyle or regularly assess the health status of employees. Organisations can establish EAPs and/or HPPs at the work place. Having access to these services would enhance employees' health and well-being. By improving and helping employees to maintain a healthy lifestyle, organisations can enhance the longevity of their employees and also increase their productivity. This would also enhance the overall productivity of the organisation because employees would be more satisfied with the organisation and would want to 'look after' the interests of the organisation in the same way. It is important to note that the EAPs and HPPs should not only focus on the physical health and well-being of employees but also should be aimed at providing a holistic approach to health and well-being.

Psychological capital is negatively related to undesirable attitudes like cynicism, turnover intentions, work stress and anxiety but are positively related to organisational citizenship behaviours (Luthans \& Youssef-Morgan, 2017). Psychological capital enhances job satisfaction, organisational commitment and psychological well-being of employees.

\section{Limitations}

The following limitations were experienced while conducting the research. Some questionnaires were not completed fully and thus had to be excluded from the study. Data had to be collected over a period of 6 weeks because of time constraints experienced by participants. This study only focussed on Namwater and Namibian Police Force and did not reach even $60 \%$ of the population. The study focussed only on specific variables; however, the researchers noted that other variables, not focussed on in the study, may also impact the relationship and outcome of the study.

\section{Recommendations}

While carrying out the study, the researchers noticed that there are a few studies conducted on this topic in the Namibian context. Thus, recommendations are given for longitudinal studies to be carried out on this topic. Perhaps, future studies can also develop an intervention and evaluate how the development of PsyCap can have a positive impact on general health and satisfaction with life.

Considering that satisfaction with life may also be impacted by non-work issues, future research could also investigate the work-home interference as part of the factors that impact satisfaction with life.

It is recommended that future studies should focus on other organisations and compare how experiences are different between organisations.

\section{Conclusion}

This study confirmed the positive relationship between PsyCap and satisfaction with life and a negative relationship between general health and satisfaction with life. This study also confirmed that hope and social dysfunction are significant predictors of satisfaction with life.

Understanding the importance and benefits of PsyCap can be beneficial for employees and organisations as employees would experience improved general health and be more satisfied with life. Organisations would be able to keep competent employees within the organisation for longer because they would be healthier and could work for more years, increase productivity and contribute to a healthy society.

Organisations need to understand their role in the lives of their employees and how a nurtured or less nurtured relationship can be instrumental or detrimental to the success of an organisation. Organisations that invest in employees are able to get back what they have invested in their employees.

\section{Acknowledgements}

The authors thank Kauyolwa N. for assisting with data collection, and the employees from both the Ministry of Safety and Security, and from Namwater.

\section{Competing interests}

The authors declare that they have no financial or personal relationships that may have inappropriately influenced them in writing this article.

\section{Authors' contributions}

I.R. was responsible for the literature review, data collection and article writing. W.R.P. was responsible for the data analysis, article writing and editing. 


\section{Funding information}

This study was supported by the University of Namibia (publishing of the article).

\section{Data availability statement}

Data sharing is not applicable to this article as no new data were created or analysed in this study.

\section{Disclaimer}

The views and opinions expressed in this article are those of the authors and do not necessarily reflect the official policy or position of any affiliated agency of the authors.

\section{References}

Aazami, S., Shamsuddin, K., Akmal, S., \& Azami, G. (2015). The relationship between job satisfaction and psychological or physical health among Malaysian working women. Penerbit Universiti Sains Malaysia, 22(4), 40-46.

Abolghasemi, A., \& Varaniyab, S.T. (2010). Resilience and perceived stress: Predictors of life satisfaction in the students of success and failure. Procedia Social and Behavioral Sciences, 5, 748-752. https://doi.org/10.1016/j.sbspro.2010.07.178

Amunkete, S.L.M.A.A. (2015). Psychological capital in Namibian state-owned enterprises: Measurement, antecedents and outcomes. Unpublished Doctora Dissertation, North-West University, South Africa.

Avey, J.B., Luthans, F., Smith, R.M., \& Palmer, N.F. (2010). Impact of positive psychological capital on employee well-being over time. Journal of Occupational Health Psychology, 15(1), 17-28. https://doi.org/10.1037/a0016998

Avey ,B.J., Wernsing, T.S., \& Mhatre, K.H. (2011). A longitudinal analysis of positive psychological constructs and emotions on stress, anxiety, and well-being. Journal
of Leadership and Organizational Studies, 18(2), 221. https://doi.org/10.1177/ of Leadership and 1548051810397368

Beins, B.C., \& McCarthy, M.A. (2012). Research methods and statistics. Boston, MA: Pearson.

Diener, E., Emmons, R.A., Larsen, R.J., \& Griffin, S. (1985). The satisfaction with life scale. Journal of Personality Assessment, 49, 71-75.

Faragher, E.B., Cass, M., \& Cooper, L.C. (2018). The relationship between job satisfaction and health: A meta-analysis. Proceedings of the Occupational and Environmental Medicine, 62, 105-112. https://doi.org/10.1136/oem.2002.006734

Fenton, S.-J., Roncancio, M.P., Sing, M., Sandhra, S., \& Carmichael, F. (2014, November 27). Workplace well-being programs and their impact on employees and their employing organisations: A scoping review of the evidence base. A collaboration between Health Exchange \& University of Birmingham. Retrieved from https://www.birmingham.ac.uk>ias.

Galanakis, M., Lakioti, A., Pezirkianidis, C., Karakasidou, E., \& Stalikas, A. (2017). Reliability and validity of the Satisfaction with Life Scale (SWLS) in a Greek sample. International Journal of Humanities and Social Studies, 5(2), 120-127.

Goldberg, D.P., \& Hillier, V.F. (1979). A scaled version of the General Health Questionnaire. Psychological Medicine, 9, 139-145.

Haidula, T. (2018). Police failed us again. The Namibian newspaper. Retrieved from https://www.namibian.com.na/179306/archive-read/Police-failed-us-again.

Haidula, L., Shino, E., Plattner, I., \& Feinstein, A. (2003). A Namibian version of the 28 item General Health Questionnaire. South African Psychiatry Review, 6, 23-25.
Hamill, S.K. (2003). Resilience and self-efficacy: The importance of efficacy beliefs and coping mechanisms in resilient adolescents. Colgate University Journal of the Sciences, 35(6), 115-146.

Hansen, A.A. (2012). Psychological capital; subjective well-being; burnout and job satisfaction amongst educators in the Umlazi region. Unpublished Master's Dissertation, University of KwaZulu-Natal, South Africa.

Herbert, M. (2011). An exploration of the relationships between psychological capital (hope, optimism, self-efficacy, resilience), occupational stress, burnout and employee engagement. Unpublished Master's Thesis, University of Stellenbosch, South Africa.

Ioannou, P., Katsikavali, V., Galanis, P., Velonakis, E., Papadatou, D., \& Sourtzi, P. (2015). Impact of job satisfaction on Greek nurses health-related quality of life. Safety and Health at Work, 6(4), 324-328. https://doi.org/10.1016/j.shaw.2015.07.010

Lu, L., Liu, L., Sui, G., \& Wang, L. (2015). The associations of job stress and organizational identification with job satisfaction among Chinese police officers: The mediating role of psychological capital. International Journal of Environment Research and Public Health, 2015(12), 15088-15099. https://doi.org/10.3390/ijerph121214973

Lucas, R.E., Diener, E., \& Suh, E. (1996). Discriminant validity of well-being measures. Journal of Personality and Social Psychology, 71(3), 616-628. https://doi org/10.1037/0022-3514.71.3.616

Luthans, F., Avolio, B., Walumbwa, F., \& Li, W. (2005). The psychological capital of Chinese workers: Exploring the relationship with performance. Management and Organization Review, 1(2), 249-271. https://doi.org/10.1111/j.1740-8784.2005. 00011.x

Luthans, F. (2002). The need for and meaning of positive organisational behaviour. Journal of Organisational Behaviour, 23(6), 695-706. https://doi.org/10.1002/ job.165

Luthans, F., Avolio, B.J., Avey, J.B., \& Norman, S.M. (2007). Positive psychological capital: Measurement and relationship with performance and satisfaction. Personnel Psychology, 60(2), 541-572. https://doi.org/10.1111/j.1744-6570.2007. 00083. $x$

Luthans, F., \& Youssef-Morgan, C.M. (2017). Psychological capital: An evidence-based positive approach. Annual Review of Organizational Psychology and Organizational
Behavior, 4(1), 339-366. https://doi.org/10.1146/annurev-orgpsych-032516-113324

Luthans, F., \& Youssef, C.M. (2004). Human, social, and now positive psychological capital management. Organizational Dynamics, 33, 143-160.

Luthans, F., Youssef, C.M., \& Avolio, B.J. (2007). Psychological capital. New York: Oxford University Press.

Mafini, C., Surujlal, J., \& Dhurup, M. (2012). Event destination factors influencing the decision to attend international sports events: A post -2010 World Cup analysis of local residents. African Journal for Physical, Health Education, Recreation and Dance, 18(1), 17-32.

Maree, J.G., \& Pietersen, J. (2007). The quantitative research process. In J. G. Maree (Ed.), First steps in research (pp. 145-153). Pretoria: van Schaik Publishers.

Mohiadin, A.M., \& Mostafai, M.A. (2012). The relationship between self-efficacy and its subscales with mental health in university students. Annals of Biological Research, 3(5), 2433-2438.

Ndokosho, J. (2006). Assessment of management approaches in a public water utility. A case study of the Namibia Water Corporation (NamWater). Unpublished Master's thesis, University of Zimbabwe, Zimbabwe.

Rode, J.C. (2004). Job satisfaction and life satisfaction revisited: A longitudinal test of an integrated model. SAGE Publications, 57(9), 1205-1230. https://doi. org/10.1177/0018726704047143

SPSS. (2016). Statistical Package for the Social Sciences. (Version 24.0) [Computer software]. Chicago, IL: SPSS Incorporated.

Sung-Mook, H., \& Effy, G. (1994). The relationship of satisfaction with life to personality characteristics. Journal of Applied and Interdisciplinary Psychology, 128(5), 547555. https://doi.org/10.1080/00223980.1994.9914912

World Health Organization (WHO). (1948). Constitution of the World Health Organization. Retrieved from https://www.who.int/governance/eb/who constitution_en.pdf. 